\title{
Combined trabeculotomy-trabeculectomy versus Ahmed valve implantation for refractory primary congenital glaucoma in Egyptian patients: a long-term follow-up
}

Hazem Helmy

Lecturer of Ophthalmology, Glaucoma and Optic Nerve Diseases Department, Glaucoma Unit, Research Institute of ophthalmology, Giza, Egypt

\section{Type of article: Original}

\begin{abstract}
Introduction: Primary congenital glaucoma (PCG) is the most common type of glaucoma in pediatric patients. The aim of this study was to compare the effectiveness of combined trabeculotomy-trabeculectomy versus Ahmed valve implantation for cases of PCG refractory to traditional incisional angle surgery (goniotomy and trabeculotomy) in the Egyptian population.

Methods: The study was conducted in the Glaucoma Clinic of the Research Institute of Ophthalmology (Egypt). The study included 66 eyes of 66 patients (in two groups) with advanced PCG who had previous failed goniotomy, trabeculotomy, or both. Group 1 included 33 patients who underwent trabeculotomy-trabeculectomy procedures; group 2 included 33 patients who underwent FP 8 Ahmed valve implantation. The main outcome measures were intraocular pressure (IOP) reduction, corneal diameter, and axial length stability in both groups. Secondary outcome measures included detection of complications in both groups.

Results: The average ages of the patients were $13.5 \pm 3.9$ months and $15.3 \pm 5.8$ months in groups 1 and 2, respectively. Ten patients (30.3\%) in group 1 and 9 patients $(27.3 \%)$ in group 2 had family histories of PCG. Positive consanguinity was present in 26 patients $(78.8 \%)$ in group 1 and in 27 patients $(81.8 \%)$ in group 2 . In group 1, the mean intraocular pressure (IOP) value decreased from $33.6 \pm 3.4 \mathrm{mmHg}$ preoperatively to $13.8 \pm 0.6$, $16.9 \pm 1.5,18.2 \pm 2.5,19.8 \pm 3.6$, and $20.2 \pm 3.1 \mathrm{mmHg}$ in the first postoperative month and after years 1, 2, 3, and 4 , respectively $(\mathrm{p}<0.001)$. In group 2 , the mean IOP value decreased from $33.4 \pm 4.5 \mathrm{mmHg}$ preoperatively to $13.3 \pm 1.1,16.3 \pm 1.6,18.1 \pm 1.0,19.1 \pm 3.5$, and $19.9 \pm 3.7 \mathrm{mmHg}$ in the first postoperative month and after years $1,2,3$, and 4 , respectively $(\mathrm{p}<0.001)$. The average number of medications decreased from $2.2 \pm 0.4$ preoperatively to $1.8 \pm 0.4$ postoperatively in group 1 , while it decreased from $2.1 \pm 0.4$ preoperatively to $1.9 \pm$ 0.3 postoperatively in group 2 . The success rate in group 1 was $97,91,76$, and $61 \%$ in years $1,2,3$, and 4 , respectively, whereas the success rate in group 2 was $97,93,81$, and $66 \%$ in years $1,2,3$, and 4 , respectively.

Conclusion: Both procedures, i.e., combined trabeculotomy-trabeculectomy and Ahmed valve implantation are suitable options in advanced PCG with failed trabeculotomy and goniotomy. Ahmed valve implanation has a better long-term survival, but trabeculotomy-trabeculectomy provides a second chance for surgical intervention.

Keywords: primary congenital glaucoma, buphthalmos, intraocular pressure, trabeculotomy-trabeculectomy, Ahmed valve implantation
\end{abstract}

\section{Introduction}

Primary congenital glaucoma (PCG) is the most prevalent type of glaucoma in the pediatric population. According to the European glaucoma society classification of childhood glaucoma, it usually presents from birth to 2 years of age (1). PCG is defined as hereditary childhood glaucoma resulting from developmental abnormality of the trabecular meshwork or the angle of anterior chamber that is not associated with other ocular, systemic congenital anomalies or acquired conditions, such as tumors, trauma, or inflammation. It is considered a diagnostic and therapeutic challenge in all centers dealing with PCG world-wide due to differences between pediatric and adult population in ocular anatomy and tissue behavior, in addition to difficult postoperative management (2-4). PCG is

\section{Corresponding author:}

Dr. Hazem Helmy, Lecturer of Ophthalmology, Glaucoma and Optic Nerve Diseases Department, Glaucoma Unit, Research Institute of ophthalmology, Giza, Egypt. E-mail: hazem.helmy@yahoo.com

Received: November 20, 2015, Accepted: January 31, 2016, Published: February 2016

iThenticate screening: January 31, 2016, English editing: February 04, 2016, Quality control: February 06, 2016

(C) 2016 The Authors. This is an open access article under the terms of the Creative Commons Attribution-NonCommercialNoDerivs License, which permits use and distribution in any medium, provided the original work is properly cited, the use is non-commercial and no modifications or adaptations are made. 
common in North Africa and is present in a more severe form than in the western world (1). The prevalence of pediatric glaucoma in the Middle East is 1:2500, whereas in consanguineous Slovakian children, it is 1:1250. The prevalence ranges between $1: 10,000$ to $1: 12,500$ in western countries $(1,5,6)$. Most patients are sporadic, but since the disease has an autosomal-recessive pattern of transmission, the incidence could increase by 500 to $1000 \%$ in certain ethnic and racial groups where consanguinity of parents predominates (7). Male subjects are more prone to develop the disease ( $70 \%$ of patients), and both eyes are simultaneously affected in the majority of patients ( $75 \%$ of patients) $(7,8)$. The main target of the treatment of pediatric glaucoma is to control the IOP surgically although medical treatment could be instated as a temporary and/or adjunctive option to maximize IOP control (9). Initial goniotomy or trabeculotomy have been detected to have high success rates (10). Trabeculotomy has a comparable long-term success rate in most patients of PCG (11). The success rate of trabeculotomy is less in advanced or latepresenting patients (12). Although goniotomy and trabeculotomy usually are associated with good success rates initially, eventually $20 \%$ of patients will require additional surgery for long-term control of IOP (13). Currently, there is no general consensus as to the preferred surgical algorithm after failure of angle surgery, and there is an extensive debate between centers that specialize in PCG practice concerning the best treatment options. Various surgical choices have been proposed for surgical treatment of PCG, including trabeculectomy with or without adjunctive anti-metabolites as mitomycin-C (MMC) (14), combined trabeculotomy-trabeculectomy (15), and glaucoma drainage devices (16). The aim of this study was to evaluate the long-term effects of combined trabeculotomy-trabeculectomy versus Ahmed valve implantation in refractory patients of PCG who had previous failed goniotomy and trabeculotomy, in addition to comparing both treatment modalities.

\section{Material and Methods}

\subsection{Setting}

This was a prospective, randomized, comparative study that included 66 eyes of 66 patients with refractory PCG who underwent incisional angle surgery and have uncontrolled IOP. The patients were randomized into two groups, each with 33 patients. The patients in group 1 had combined trabeculotomy-trabeculectomy, whereas the patients in group 2 had Ahmed valve implantation. All surgeries were done by the same surgeon. The patients were recruited from the glaucoma clinic of the Research Institute of Ophthalmology (R.I.O.) in Egypt from 2011-2012. Patients underwent full ophthalmologic examination while under general anesthesia in a previous session prior to surgery. Examination included corneal diameter measurement with caliper, IOP measurement with Perkin's applanation tonometer within the first few minutes of anesthesia, indirect ophthalmoscopy, and refraction in patients were corneal clarity and red reflex allowed examination and obtaining accurate results. Direct gonioscopy was done with Zeiss gonioscopy lens (Ocular 1.5X, Bellevue, Washington, USA). The axial length was measured with A-scan ultrasound (SONOMED EZ-SCAN COMBOA-SCAN/B-SCAN) under sedation with chloral hydrate. Exclusion criteria were patients with other types of primary and secondary pediatric glaucomas and previous ocular surgery except incisional glaucoma surgery (trabeculotomy or trabeculectomy).

\subsection{Surgical technique}

The surgical techniques included combined trabeculotomy-trabeculectomy and Ahmed valve implantation (Ahmed valve implantation model fp8 (New World Medical, Inc., Rancho Cucamonga, CA, USA)). In the first technique, following general anesthesia, superior rectus bridle suture was placed with 6-0 silk suture. A fornix-based conjunctival flap was fashioned, and homeostasis was secured with bipolar cauterization. A $4 \times 2.5-\mathrm{mm}$ partial thickness scleral flap was dissected into approximately $1 \mathrm{~mm}$ in clear cornea. A $2 \times 2-\mathrm{mm}$ inner trabeculectomy groove was made. A radial incision was made across the inner trabeculectomy site approximately $2 \mathrm{~mm}$ from the limbus. The incision was done at the junction between the white and bluish transitional zone of the sclera, which coincides with the site of Shlemm's canal. Then, Shlemm's canal was entered. Successful entry into the canal was evidenced by gush of an aqueous liquid and/or blood. Then, trabeculotomy was performed using the internal arm of Harm's trabeclotome probe, first to the left then to the right to perform an incision along 100-120 of circumference. The pre-marked $2 \times 2-\mathrm{mm}$ inner block tissue comprising the trabecular meshwork and scleral spur was excised with Vannus scissors and peripheral iridectomy was performed with a base of at least $2 \mathrm{~mm}$. Postoperatively, all patients received steroids, antibiotics, and cycloplegic drops daily. The cycloplegic drops were stopped at 7-10 days, the antibiotic drops were stopped at two weeks after surgery, and the steroid drops were tapered off gradually over a period of 4-8 weeks. In the second surgical technique, following general anesthesia, corneal traction bridle suture was applied. A fornix-based conjunctival flap was fashioned. Tenon's capsule was dissected to create a pocket to allow insertion of the plate of the valve 6-8 $\mathrm{mm}$ posterior to the limbus. The valve was primed with balanced salt saline. The plate was fixed to the sclera with two $8 / 0$ black nylon sutures. A 4-mm radial scleral tunnel was created with a 23-gauge needle towards the limbus. An anterior chamber paracentesis wound was created at the peripheral 
cornea and 1\% sodium hyaluronate was injected to prevent collapse of the anterior chamber after sclerotomy was done. The tube was shortened to the desired length with its sharp bevel facing anteriorly to allow 2-3 mm of tube in the anterior chamber. The tube of the implant entered the anterior chamber parallel to the iris plane through the radial sclerotomy track. The tube was fixed to the sclera with $9 / 0$ black nylon suture. The conjunctiva were closed with $8 / 0$ polyglactin (Vicryl; Ethicon Ltd.) sutures. The sodium hyaluronate in the anterior chamber was removed as much as possible through the paracentesis site. Postoperatively, all patients received steroids and antibiotic drops daily. The antibiotic drops were stopped at two weeks postoperatively, and the steroid drops were tapered gradually over 4-8 weeks.

\subsection{Patients' Follow-up Regimen}

The type of glaucoma surgery was recorded in addition to intra-operative and postoperative complications and their management. Postoperative follow-up visits were recorded at 1, 3, and 6 months and thereafter every six months for 48 months. At each visit, a full ophthalmological examination was done under general anesthesia that included corneal diameter measurement, Perkins' applanation tonometry, indirect ophthalmoscopy, and refraction.

\subsection{Success criteria}

Complete success criteria included postoperative IOP value $>6$ and $\leqslant 21 \mathrm{mmHg}$ without additional medical or surgical treatment, stable corneal diameter, decreased corneal edema, improved corneal clarity, and stable or reversed cup disc/ratio. Qualified success was considered if the aforementioned IOP range could be achieved with additional use of anti-glaucoma treatment or surgical valve revision. Failure was considered if such IOP range could not be reached even with maximal tolerated medical treatment, or if subsequent glaucoma surgery was needed to control IOP. All procedures and follow-up visits were done at the Research Institute of Ophthalmology (R.I.O)Ministry of Scientific Research, Egypt. The study was performed in accordance with the tenets of the Declaration of Helsinki of 1975 (1983 revision). The Research Committee of the Research Institute of Ophthalmology approved the protocol of the study. All patients' parents received a thorough explanation of the procedures entailed in the study and signed an informed consent prior to enrollment.

\subsection{Statistical analysis}

The data were statistically described in terms of mean standard deviation ( $\mathrm{SD})$, median and range, or frequencies (number of patients) and percentages when appropriate. Comparison of numerical variables between the study groups was done using the Student's t-test for independent samples. Within group comparison of numerical variables was done using the paired t-test. For comparing categorical data, the chi-squared ( 2 ) test was performed. Exact test was used instead when the expected frequency was less than 5. Correlation between various variables was done using Pearson's moment correlation equation for linear relation in normally distributed variables and Spearman's rank correlation equation for non-normal variables/non-linear monotonic relation. The p-values less than 0.05 were considered statistically significant. All statistical calculations were done using SPSS software, version 15, for Microsoft Windows (SPSS, Inc., Chicago, Illinois, USA).

\section{Results}

The mean age of patients was $13.5 \pm 3.9$ months in group 1 and $15.3 \pm 5.8$ months in group 2 . In group 1,17 patients $(51.5 \%)$ were females; whereas in group 2, 17 patients $(51.5 \%)$ were males. The number of previous operations was $2.0 \pm 0.3$ in group 1 and $1.9 \pm 0.4$ in group 2 . The family history of PCG was positive in 10 patients $(30.3 \%)$ in group 1 and in 9 patients $(27.3 \%)$ in group 2. Positive consanguinity was present in 26 patients $(78.8 \%)$ in group 1 and in 27 patients $(81.8 \%$ ) in group 2 (Table 1). Preoperative mean horizontal corneal diameter was 14.5 $\pm 0.8 \mathrm{~mm}$ in group 1 , and it was $14.4 \pm 0.7 \mathrm{~mm}$ in group 2. Preoperative mean vertical corneal diameter was $13.8 \pm$ $0.7 \mathrm{~mm}$ in group 1, and it was $13.7 \pm 0.7 \mathrm{~mm}$ in group 2. The mean values of horizontal and vertical corneal diameters remained stable throughout the study in both groups (Table 1). Preoperative mean cup/disc ratio was 0.89 \pm 0.01 in group 1 versus $0.90 \pm 0.01$ in group 2 . That ratio remained stable throughout the follow-up period in both groups (Table 1). The average number of medications decreased from $2.2 \pm 0.4$ preoperatively to $1.8 \pm 0.4$ in group 1 , and from $2.1 \pm 0.4$ preoperatively to $1.9 \pm 0.3$ postoperatively in group 2 (Table 1). The mean preoperative IOP was $33.6 \pm 3.4 \mathrm{mmHg}$ in group 1, and it was decreased to $17 \pm 1.5,18.2 \pm 2.5,20 \pm 3.6$, and $20.2 \pm 3 \mathrm{mmHg}$ in years 1, 2, 3, and 4, respectively. The difference between preoperative and postoperative IOP was statistically significant $(\mathrm{p}<0.0001)$. The mean preoperative IOP was $33.4 \pm 4.5 \mathrm{mmHg}$ in group 2 , and it was decreased to 16.3 $\pm 1.6,18.1 \pm 1.0,19.1 \pm 3.5$, and $19.9 \pm 3.7 \mathrm{mmHg}$ in years $1,2,3$, and 4 , respectively. The difference between preoperative and postoperative IOP was statistically significant $(\mathrm{p}<0.0001)$ (Tables 1 and 2) (Figure 1). 
Table 1. Preoperative Characteristics

\begin{tabular}{|l|l|l|l|}
\hline \multicolumn{2}{|l|}{ Variables } & Group 1 & Group 2 \\
\hline Age & $13.5 \pm 3.9$ & $15.3 \pm 5.2$ \\
\hline \multirow{2}{*}{ Gender } & Male & $17(51.5 \%)$ & $16(48.5 \%)$ \\
\cline { 2 - 4 } & Female & $16(48.5 \%)$ & $17(51.5 \%)$ \\
\hline Family History & $10(30.3 \%)$ & $9(27.3 \%)$ \\
\hline Consanguinity & $26(78.8 \%)$ & $27(81.8 \%)$ \\
\hline IOP & $33.6 \pm 3.4$ & $33.42 \pm 4.5$ \\
\hline Axial Length & $25.9 \pm 0.9$ & $26.00 \pm 0.7$ \\
\hline \multirow{2}{*}{\begin{tabular}{l|l|} 
Corneal \\
Diameter
\end{tabular}} & V & $14.5 \pm 0.8$ & $14.4 \pm 0.71$ \\
\hline \multicolumn{2}{|l|}{ Anti-glaucoma Medications } & $13.8 \pm 0.7$ & $13.7 \pm 0.7$ \\
\hline Number of Surgeries & $2.2 \pm 0.4$ & $2.1 \pm 0.4$ \\
\hline CD Ratio & $2.0 \pm 0.3$ & $1.9 \pm 0.4$ \\
\hline Family history & $0.9 \pm 0.01$ & $0.9 \pm 0.01$ \\
\hline \multicolumn{2}{|l|}{ consanguinity } & $10(30.3 \%)$ & $9(27.7 \%)$ \\
\hline
\end{tabular}

Table 2. Postoperative Characteristics

\begin{tabular}{|l|l|l|l|}
\hline \multicolumn{2}{|l|}{ Variables } & Group 1 & Group 2 \\
\hline IOP & $20.2 \pm 3.1$ & $19.9 \pm 3.7$ \\
\hline \multirow{2}{*}{ Corneal Diameter } & H & $14.5 \pm 0.8$ & $14.4 \pm 07$ \\
\cline { 2 - 4 } & V & $13.8 \pm 0.7$ & $13.7 \pm 0.7$ \\
\hline Axial Length & $25.8 \pm 0.9$ & $26.00 \pm 0.7$ \\
\hline \multicolumn{2}{|l|}{ Anti-glaucoma Medications } & $1.8 \pm 0.4$ & $1.9 \pm 0.3$ \\
\hline
\end{tabular}

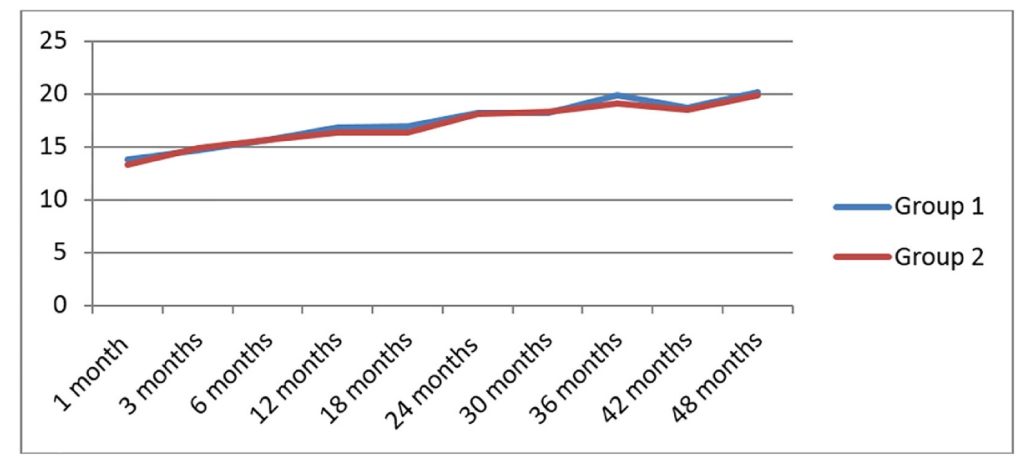

Figure 1. Postoperative IOP in both groups during the follow-up period

Mean preoperative axial length was $26 \pm 0.9 \mathrm{~mm}$ in group 1 . Mean postoperative axial length was $26 \pm 0.9,26 \pm 0.8$, $26 \pm 0.8$, and $26 \pm 0.9 \mathrm{~mm}$ in years $1,2,3$, and 4 , respectively. The difference between preoperative and postoperative axial length was insignificant $(\mathrm{p}=0.23,0.15$, and 0.47 in years 1,2 , and 3 , respectively. Then, it became significant in the fourth year $(\mathrm{p}=0.008)$ (Tables 1 and 2). Mean preoperative axial length was $26.00 \pm 0.7$ $\mathrm{mm}$ in group 2. Mean postoperative axial length was $26 \pm 0.7,26 \pm 0.7,26 \pm 0.7$, and $26 \pm 0.7 \mathrm{~mm}$ in years $1,2,3$, and 4 , respectively. The difference between pre- and post-operative axial length was insignificant as $p=0.38,0.22$, 0.007 in years 1,2 , and 3, respectively, while it was significant in the fourth year $(\mathrm{p}<0.0001)$ (Tables 1 and 2$)$. Postoperative complications included hyphema in 15 patients (45.4\%) in group 1 and in 5 patients $(15.1 \%)$ in group 2. Hyphema resolved spontaneously within 5 to 7 days without any additional treatment. Lost AC occurred in 3 patients $(9 \%)$ in group 1 and in 2 patients $(6 \%)$ in group 2 that required $\mathrm{AC}$ reformation with saline and viscoelastic injection. Choroidal effusion appeared in 3 patients (9\%) in group 1 and in 2 patients (6\%) in group 2 that resolved spontaneously with systemic and topical steroids plus atropine eye drops. Tenon's encapsulated bleb appeared in 2 patients $(6 \%$ ) (Table 3). The long-term probability success rate was $97,91,76$, and $61 \%$ in years 1,2 , 3 , and 4, respectively, in group 1 , and $97,93,81$, and $66 \%$ in years $1,2,3$, and 4, respectively, in group 2 (Table 4). 
Table 3. Complications

\begin{tabular}{|l|l|l|}
\hline Variables & Group 1 & Group 2 \\
\hline Hyphaema & $15(45.4 \%)$ & $5(15.1)$ \\
\hline Lost AC & $3(9.09 \%)$ & $2(6.06 \%)$ \\
\hline Choroidal Effusion & $3(9.09 \%)$ & $2(6.06 \%)$ \\
\hline
\end{tabular}

Table 4. The Long term probability success rate

\begin{tabular}{|l|l|l|}
\hline \multirow{2}{*}{ Years after surgery } & \multicolumn{2}{|l|}{ Success rate } \\
\cline { 2 - 3 } & Group 1 & Group 2 \\
\hline $1^{\text {st }}$ year & $97 \%$ & $97 \%$ \\
\hline $2^{\text {nd }}$ year & $91 \%$ & $93 \%$ \\
\hline $3^{\text {rd }}$ year & $76 \%$ & $81 \%$ \\
\hline $4^{\text {th }}$ year & $61 \%$ & $66 \%$ \\
\hline
\end{tabular}

If you don't care for the last four suggested changes, you could use first, second, third, and fourth.

\section{Discussion}

The main aim for surgical management of PCG is reduction of IOP9. Therefore, surgical procedures mainly were rated by their efficacy, safety, and feasibility. The first line of surgery for PCG is angle incisional surgery, namely, goniotomy and trabeculotomy. Although initial goniotomy or trabeculotomy revealed comparable success rates (10), refractory pediatric glaucoma continues to impose a therapeutic challenge because, despite the high initial success rate of incisional angle surgery, almost $20 \%$ of angle procedures eventually fail. A trabeculectomy with or without adjunctive anti-fibrosis therapy, glaucoma drainage devices or cyclo-destructive procedures are considered the second line of treatment. Recent studies of trabeculectomy suggest that MMC application is associated with high risk of late-onset complications related to infection $(13,16)$. Trabeculotomy-trabeculectomy and glaucoma drainage devices implantation may represent a sound choice for these patients. Hoskins et al. classified PCG anatomically based on gonioscopy into three types, i.e., trabeculo-dysgenesis, iridotrabeculo-dysgenesis, and corneotrabeculodysgenesis, and they recommended trabeculotomy-trabeculectomy as a primary procedure for the last two groups due to the low success rate of goniotomy and trabeculotomy in these types. The probability success rate was $90 \%$ for the first group, while $30 \%$ was the success rate for both the second and third groups (17, 18). Combining trabeculotomy with trabeculectomy provides multiple additions over conventional surgeries, which aggravate the result in controlling IOP and was not achieved with these surgeries. It provides two ways for aqueous outflow as trabeculotomy creates a direct communication between the anterior chamber and Schlemm's canal, while sub-scleral trabeculectomy by-passes the episcleral venous system. It also reserves the option for conversion to trabeculectomy in case of inability to identify the Schlemm's canal properly (19). In the present study, the authors tried to detect the long-term effect of trabeculotomy-trabeculectomy in comparison with Ahmed valve implantation in management of refractory PCG. The results of the present study showed that the mean IOP value decreased from $33.6 \pm 3.4$ preoperatively to $13.8 \pm 0.6,16.9 \pm 1.5,18.2 \pm 2.5,19.9 \pm 3.6$, and $20.2 \pm 3.1$ in the first postoperative month and in years $1,2,3$, and 4, respectively, in group $1(\mathrm{p}<0.001)$. Moreover, we had a primary success rate of 97, 91, 76, and $61 \%$ in years $1,2,3$, and 4 , respectively. The findings of the present study are congruous with a long-term study done by Mandal (20) that included 299 patients with PCG in which primary combined trabeculotomytrabeculectomy was performed. Those authors demonstrated that the mean IOP value decreased from $26.6 \pm 6.2$ $\mathrm{mmHg}$ to $14.4 \pm 4.9 \mathrm{mmHg}$. The probability of success was 94.4, 92.1, 86.7, 79.4, 72.9, and 63.1\% at years $1,2,3$, 4, and 6, respectively. In another study by Mandal et al., (21) they aimed at detecting the safety and efficacy of simultaneous bilateral primary combined trabeculotomy-trabeculectomy for developmental glaucoma. The authors reported probability of success rates of $90.9,88$, and $69.3 \%$ at years 1,2 , and 3 , respectively. The probability of success rate of the present study lies between the probability of success rates of the above two studies introduced by Mandal and Mandal et al. $(20,21)$; this could be explained by the fact that the first study included cases of PCG but trabeculotomy-trabeculectomy was done as a primary procedure, while the second study dealt with cases with developmental glaucoma. In another study by Elder (19), comparisons were made between combined trabeculotomy-trabeculectomy and primary trabeculectomy alone for management of congenital glaucoma, and the authors reported a $72 \%$ probability of success rate in cases that underwent primary trabeculectomy. However, the probability of success rate in cases treated with the combined procedure was $93.5 \%$. The follow-up period of that study was only 24 months. Contrary to our results, Al-Hamzi et al. (22) reported a success rate of $70 \%$ in 1 or 3 
years for combined trabeculotomy -trabeculectomy with MMC performed in severe cases of congenital glaucoma in which the IOP exceeded $35 \mathrm{mmHg}$ and in which the corneal diameter was larger than $14.5 \mathrm{~mm}$. The higher success rate in the present study in comparison with the study done by El Hamzi et al. (22) may be related to the inclusion criteria that required only cases with PCG that had failed one- or two-incisional angle surgeries. In our cases, the site of trabeculotomy -trabeculectomy was elected in an area with virgin conjunctiva that may have helped in increasing the success rate. Glaucoma drainage implants have been used successfully for the treatment of refractory glaucoma. One of the common early postoperative complications is over-drainage producing shallow AC, hypotony, and choroidal detachment. Ahmed glaucoma valve implantation has a lower rate of hypotony due to built-in venture valve that offers resistance to the aqueous outflow (23). In our study, the mean IOP decreased from $33.4 \pm 4.5$ preoperatively to $13.3 \pm 1.1,16.3 \pm 1.6,18.1 \pm 1.0,19.1 \pm 3.5$, and $19.9 \pm 3.7$ in the first postoperative month and in years $1,2,3$, and 4 , respectively, in group $2(\mathrm{p}<0.001)$. In addition, FP8 Ahmed valve implantation had success rates of $97,93,81$, and $66 \%$ in years $1,2,3$, and 4 , respectively.

Our findings in terms of success rate are in accordance with the $92 \%$ success rate achieved by O'Malley et al. (24). Those authors studied 38 eyes with congenital glaucoma and 41 eyes with aphakic glaucoma. Their reported followup periods were 5.5 and 3.5 years in the first and second groups, respectively. In that study, the preoperative mean IOP value was $29 \mathrm{mmHg}$ in the first group, and it was decreased to $14 \mathrm{mmHg}$ postoperatively; the mean preoperative IOP was $36 \mathrm{mmHg}$ in the second group, and it was decreased to $15 \mathrm{mmHg}$ post-operatively. Contrary to our findings, Ahmed valve implantation had a success rate of $54.5 \%$ in a long-term comparison study between single stage Barveldet and Ahmed glaucoma implants in pediatric glaucoma (25). That study included 11 cases for Ahmed valve implantation in comparison with 20 patients for Barveldet valve implantation. The difference between that study and ours was in the material used in the synthesis of the Ahmed valve. Those authors used a PMMA S2 valve, and we used a silicone-plated implant (FP8) in our study. Moreover, the present study included only cases of PCG; whereas El Gendy et al. (25) included all pediatric refractory glaucoma patients (aphakic, neovascular, anirida, steroid induced, retinopathy of prematurity, congenital hereditary endothelial dystrophy and microphthalmia). In addition, the number of patients in our study was greater than the number in the study of El Gendy et al. (25). A study by Ayyala et al. (26) concluded that in experimental models of glaucoma drainage devices silicon was associated with less inflammation and fibrosis than polypropylene. Similarly, Khan et al. (27) compared the 2-year probability of survival rate of polypropylene and silicone Ahmed glaucoma valves. The study included 31 patients who received a polypropylene Ahmed glaucoma valve and 11 patients who received a silicone Ahmed glaucoma valve. The average survival time was significantly longer, i.e., $23.4 \pm 1.6$ months, for the silicone group; it was 19.1 \pm 1.5 months for the polypropylene group. The cumulative probabilities of survival were 90.9 and $54.8 \%$ in each group, respectively. In terms of complications, hyphema was detected in $5(15 \%)$ of our patients, but it resolved spontaneously within a few days; according to Ayyala et al. (28) hyphema is a common postoperative complication with Ahmed valve implantation. Another reported complication that did not occur in our study was late tube malposition $(29,30)$. According to Murgacz et al. (31), this complication can be related to inadequate surgical maneuver or fibrous tissue contracture around the valve. Relative elastic recoil may occur when the IOP becomes normalized. These authors assumed that tube rotation occurred around the scleral spur, and the tube took a more anterior position closer to the corneal endothelium. They also concluded that placing these tubes closer to the iris may compensate for this anterior rotation. Morad et al. (16) detected that the probability of success rate of Ahmed valve implantation decreases with time. In their study, the success rates were $93,86,71$, and $45 \%$ at $1,2,3$, and 4 years, respectively (16). The higher success rate reported in our study could be attributed to the fact that all our patients had a virgin upper conjunctiva as all previous procedures were approached temporally. In addition, the ethnicity of the studied population probably played a role in improving our success rate. All patients in our study were Egyptians. Ou et al. (32) demonstrated less favorable surgical outcome associated with Hispanic ethnicity and female gender. The presence of hypertensive phase defined as IOP $\geqslant 21 \mathrm{mmHg}$ during the first 3 months after surgery was seen in 8 eyes $(24.2 \%)$ and that resolved in all patients. This is comparable to previous studies that showed a hypertensive phase in $25-82 \%$ in adults (33). However, in pediatric glaucoma, Chen et al. (34) found 25$40.4 \%$ having hypertensive phase, demonstrating that the incidence is lower in children. Comparison between the two groups in the current study concerning the probability of success rate indicated that both groups have the same probability of success rates in the first year. The probability of success rate decreased along the long-term follow up period in both groups but became higher in Ahmed valve group in comparison with trabeculotomy-trabeculectomy group. IOP reduction rate and number of anti-glaucoma treatment postoperatively were similar in both groups. To the best of our knowledge, this is the first study that has compared trabeculotomy-trabeculectomy and Ahmed valve implantation in refractory PCG. Contrary to our results, Pakravan et al. (35) reported success rates of 87 and $73 \%$ after a mean follow up time of 16.3 and 13.1 months with Ahmed valve and trabeculectomy with MMC, 
respectively. The IOP reduction rate and the number of glaucoma medications needed were similar in the two groups. The findings of those authors were consistent with our study as the long term probability of success rate of Ahmed valve implantation was higher than trabeculectomy, and they have the same effect in IOP and anti-glaucoma treatment reduction. They differ with the current study in the probability of success rate after one to 18 months follow-up being lower. This may be explained by the limited number of patients included in Pakravan's study and the specific type of patients included in the study, since all of them had aphakic pediatric glaucoma, and they only did trabeculectomy. All failed patients of trabeculotomy-tyrabeculectomy group underwent Ahmed valve implantation or cyclophotocoagulation and eventually had their IOP controlled. All failed patients of Ahmed valve implantation group, except two patients who needed revision for encapsulated bleb, required multiple cyclophotoablation to control their IOP. Although the trabeculotomy-trabeculectomy procedure has a lower longterm success rate than Ahmed valve implantation, it offers a chance to implant glaucoma drainage device as a last resort before the cyclo-ablation procedure.

\section{Conclusions}

Trabeculotomy -trabeculectomy and Ahmed valve implantation are suitable options in advanced buphthalmos with failed trabeculotomy and goniotomy. Ahmed valve implanation has a better long-term survival, but trabeculotomytrabeculectomy provides a second chance for surgical intervention.

\section{Acknowledgments:}

The author thanks the Research Institute of Ophthalmology for helping us in this research.

\section{Conflict of Interest:}

There is no conflict of interest to be declared.

\section{References}

1) Jaafar MS. Care of infantile glaucoma patients. Ophthalmology Annual, Raven Press, New York, NY, USA, 1998.

2) Ho CL, Walton DS. Primary congenital glaucoma. J Pediatr Ophthalmol Strabismus. 2004; 41: 271-88. PMID: 15478740.

3) Taylor RH, Ainsworth JR, Evans AR, Levin AV. The epidemiology of pediatric glaucoma: the Toronto experience. J AAPOS. 1999; 3: 308-15. doi: 10.1016/S1091-8531(99)70028-5, PMID: 10532577.

4) Ou Y, Caprioli J. surgical management of pediatric glaucoma. Dev Ophthalmol. 2012; 50: 157-72. doi: 10.1159/000334798, PMID: 22517182.

5) Mandal A, Chakrabarti D. Update on congenital glaucoma. Indian J ophthalmol. 2011; 59(1): 148-57. doi: 10.4103/0301-4738.73683, PMID: 21150027, PMCID: PMC3038500.

6) Khan AO. Genetics of primary glaucoma. Curr Opin Ophthalmol. 2011; 22(5): 347-55. doi: 10.1097/ICU.0b013e32834922d2, PMID: 21730848.

7) European Glaucoma society, Terminology and guidelines for glaucoma, European glaucoma society, 4th edition, 2014.

8) Papadopoulos M, Cable N, Rahi J, Khaw PT. BIG eye study investigators. The British infantile and childhood glaucoma (BIG) eye study. Invest Ophthalmol Vis Sci. 2007; 48: 4100-6. doi: 10.1167/iovs.061350, PMID: 17724193 .

9) Al sheikheh A, Klink J, Klink T. Long term results of surgery in childhood glaucoma. Arch Clin Exp Ophthalmol. 2007; 245: 195-203. doi: 10.1007/s00417-006-0415-2, PMID: 16983524.

10) Yalvac IS, Satana B, Suveren A, Eksioglu U, Duman S. Success of trabeculotomy in patients with congenital glaucoma operated on within 3 months of birth. Eye. 2007; 21: 459-64.

11) Cai Y, Li MY, Shen YY, Liu LN. Long-term effect of trabeculotomy on primary congenital glaucoma. Zhongua Yan Ke Za Zhi. 2004; 40(11): 733-36. PMID: 15634478.

12) Correlation between surgical success rate and severity of congenital glaucoma. Br J Ophthalmol. 2005; 89(4): 449-53. doi: 10.1136/bjo.2004.047761, PMID: 15774922, PMCID: PMC1772605.

13) Tanimoto SA, Brandt JD. Options in pediatric glaucoma after angle surgery has failure. Curr Opin Ophthalmol. 2006; 17(2): 132-7. doi: 10.1097/01.icu.0000193091.60185.27, PMID: 16552247.

14) Rodrigues AM, Junior AP, Montezano FT, de Arruda Melo PA, Parata JJ. Comparison between results of trabeculectomy in primary congenital glaucoma with and without the use of mitomycin C. J Glaucoma. 2004; 13(3): 228-32. doi: 10.1097/00061198-200406000-00010, PMID: 15118468. 
15) Mullaney PB, SelleckC, Al-Awad A. Combined trabeculectomy and trabeculotomy as an initial procedure in uncomplicated congenital glaucoma. Arch Ophthalmol. 1999; 1117; 457-60. doi: 10.1001/archopht.117.4.457.

16) Morad Y, Donaldson CE, Kim YM, Abdolell M, Levin AV. The Ahmed drainage implant in the treatment of pediatric glaucoma. Am J Ophthalmol. 2003; 135: 821-9. doi: 10.1016/S0002-9394(02)02274-2, PMID: 12788122.

17) Hoskins HD Jr, Shaffer RN, Hetherington J. Anatomical classification of the developmental glaucoma. Arch Ophthalmol. 1984; 102: 1331-6. doi: 10.1001/archopht.1984.01040031081030, PMID: 6477252.

18) Luntz MH. The choice of surgical procedure in congenital infantile, and juvenile glaucoma. J Ophthalmic Nurs Technol. 1990; 9: 100-1. PMID: 2366246.

19) Elder MJ. Combined trabeculotomy -trabeculectomy compared with primary trabeculectomy for congenital glaucoma. Br J Ophthalmol. 1994; 78: 745-8. doi: 10.1136/bjo.78.10.745, PMID: 7803349, PMCID: PMC504926.

20) Mandal AK. Long term surgical and visual outcomes in indian children with developmental glaucoma operated on within 6 months. Ophthalmology. 2004; 111: 283-90. doi: 10.1016/j.ophtha.2003.05.027, PMID: 15019376.

21) Mandal AK, Bhatia PG, Gothwal VK, Reddy VM, Siramulu P, Prasad MS, et al. Safety and efficacy of simultaneous bilateral primary combined trabeculotomy-trabeculectomy for developmental glaucoma in India. Indian J Ophthalmol. 2002; 50: 13-9. PMID: 12090080.

22) Al-Hamzi A, Awad A, Zwaan J, et al. Correlation between surgical success rate and severity of congenital glaucoma. Br J Ophthalmol. 2005; (4): 449-53.

23) Lim KS, Allan BDS, Liod AW, Muir A, Khaw PT. Glaucoma drainage devices; past, present and future. Br J Ophthalmol. 1998; 82: 1083-9. doi: 10.1136/bjo.82.9.1083, PMID: 9893602, PMCID: PMC1722728.

24) O,Malley Schotthoefer E, Yanovitch TL, Freedman SF. Aqueous drainage device surgery in refractory pediatric glaucoma. Long-term outcomes. J AAPOS. 2008; 12(1): 33-9. doi: 10.1016/j.jaapos.2007.07.002, PMID: 17942353.

25) El Gendy NMS, Song JC. Long term comparison between single stage Baerveldt and Ahmed glaucoma implants in pediatric glaucoma. Saudi J Ophthalmol. 2012; 26(3): 323-6. doi: 10.1016/j.sjopt.2012.06.003, PMID: 23961013, PMCID: PMC3729322.

26) Ayyala RS, Michelini-Norris B, Flores A, Haller E, Margo CE. Comparison of different biomaterials for glaucoma drainage devices: part 2. Arch Ophthalmol. 2000; 118(8): 1081-4. doi: 10.1001/archopht.118.8.1081, PMID: 10922202.

27) Khan AO. AL mobarak FA. Comparison of polypropylene and silicon Ahmed valve survival 2 years following implantation in the first 2 years of life. $\mathrm{Br} \mathrm{J}$ Ophthalmol. 2009; 93(6): 791-4. doi: 10.1136/bjo.2008.151258, PMID: 19279020.

28) Ayyala RS, Zurakowski D, Smith JA, Monshizadeh R, Netland PA, Richards DW, et al. A clinical study of the Ahmed glaucoma valve implant in advanced glaucoma. Ophthalmol. 1998, 105: 1968-76. doi: 10.1016/S0161-6420(98)91049-1, PMID: 9787371.

29) Coleman AL, Smyth RG, Wilson MR, Tam M. Initial clinical experience with the Ahmed valve implantation pediatric patients. Arch Ophthalmol. 1997, 115: 186-91. doi: 10.1001/archopht.1997.01100150188007, PMID: 9046253.

30) Englert JA, Freedman SF, Cox TA. The Ahmed valve in refractory pediatric glaucoma. Am J Ophthalmol. 1999, 127: 34-42. doi: 10.1016/S0002-9394(98)00292-X.

31) Mrugacz M, Bryl A. Ahmed glaucoma valve implantation in the surgical treatment of traumatic glaucoma case report. J Ophthalmic Cli Res. 2015; 2: 004.

32) OU Y, Yu, Law SK, Coleman AL, Caprioli J. Outcomes of Ahmed glaucoma valve implantation in children with primary congenital glaucoma. Arch Ophthalmol. 2009; 127(11): 1436-41. doi: 10.1001/archophthalmol.2009.267, PMID: 19901208.

33) Nouri-Madhafi K, Caprioli J. Evaluation of the hypertensive phase after insertion of the Ahmed glaucoma valve. Am J Ophthalmol. 2003; 1001-8. doi: 10.1016/S0002-9394(03)00630-5.

34) Chen TC, Bhatia LS, Walton DS. Ahmed valve surgery for refractory pediatric glaucoma: a report of 52 eyes. J Pediatr Ophthalmol Strabismus. 2005; 42: 274-83. PMID: 16250216.

35) Pakravan M, Homayoon N, Shahin Y, Ali Reza BR. Trabeculectomy with mitomycin C versus Ahmed glaucoma implant with mitomycin $\mathrm{C}$ for treatment of pediatric aphakic glaucoma. J Glaucoma. 2007; 16(7): 631-6. doi: 10.1097/IJG.0b013e3180640f58, PMID: 18091183. 